\title{
GROUNDING AND METAPHYSICAL EXPLANATION
}

\author{
NAOMI THOMPSON
}

Attempts to elucidate grounding are often made by connecting grounding to metaphysical explanation, but the notion of metaphysical explanation is itself opaque, and has received little attention in the literature. We can appeal to theories of explanation in the philosophy of science to give us a characterization of metaphysical explanation, but this reveals a tension between three theses: that grounding relations are objective and mindindependent; that there are pragmatic elements to metaphysical explanation; and that grounding and metaphysical explanation share a close connection. Holding fixed the mind-independence of grounding, I show that neither horn of the resultant dilemma can be blunted. Consequently, we should reject the assumption that grounding relations are mindindependent.

This paper is about the grounding relation and the connection between grounding and explanation. Grounding is a relation of non-causal ontological dependence, a metaphysical determination relation which obtains between entities of various ontological categories including facts, properties, states of affairs, and actual concrete objects. Though discussion of grounding has become widespread, much of the literature about grounding is devoted to arriving at a proper characterization of the notion. Difficulties arise because grounding is taken to be a metaphysical primitive-it resists reductive analysis. This leaves grounding vulnerable to the charge that it has no distinctive content, or no useful role to play. In response (as well as pre-emptively) grounding has been defended by appeal to intuitive examples of grounding, by highlighting the usefulness of a grounding relation, and by connecting grounding to other more familiar metaphysical notions. In particular, grounding has been connected to the notion of explanation (Fine 200I, 20I2; Schaffer 2009; deRosset 20I0; Audi 20I2; Raven 2013).

To say that $x$ grounds $y$ is to provide some kind of explanation of $y$ in terms of $x$. Defenders of grounding usually insist that the 
explanation on offer is somehow 'metaphysical'. For example, Fine (20I2, p. 37) introduces the idea of ontological ground as 'a distinctive kind of metaphysical explanation, in which explanans and explanandum are connected ... through some constitutive form of determination'. Unfortunately, the precise sense in which the relevant kind of explanation is distinctive remains somewhat opaque. I will argue that attempts to specify a notion of metaphysical explanation useful for elucidating grounding are bound to fail.

Articulating the Problem. Here are three independently plausible, 10 but jointly problematic, theses about grounding and explanation:

(i) There is a tight connection between grounding and metaphysical explanation.

(ii) Metaphysical explanation has agent-relative features.

(iii) Grounding is objective and mind-independent.

If grounding is objective and mind-independent, but metaphysical explanation has pragmatic or agent-relative features, then it seems the connection between grounding and explanation can't be as close as the literature suggests. If the connection is to be maintained along with the objectivity of grounding, then metaphysical explanation must be similarly objective and mind-independent. Alternatively, grounding, like metaphysical explanation, must have minddependent features. Call this tension 'the grounding-explanation problem'.

Why believe the three theses of the triad? I have said something about (i) already; it is a cornerstone of the grounding literature. The same is true of (iii). Grounding is supposed to be a paradigmatically worldly relation: grounding relations limn the structure of reality. The only controversial claim amongst the three theses is the second: that metaphysical explanation has pragmatic or agent-relative features. Let's consider the problem in the form of a dilemma. Assume (iii). We must now deny either (i) or (ii). Since we've noted that (ii) is the more controversial of the two, we'll start there. 
First Horn: Metaphysical Explanation. Why accept that explanation, and in particular metaphysical explanation, has pragmatic features? A quick survey of the literature on ordinary explanation (which I do not have the space to rehearse here) reveals that all the major theories of explanation build features of the explanationseeker into their account of successful explanation. Explanations are successful when they move the explanation-seeker to expect or understand the explanandum on the basis of the explanans. Expectation and understanding are agent-relative notions.

Two things remain thus far unexplained. First, why is it successful explanation, rather than mere explanation, that we are interested in? Second, why should we think that metaphysical explanation has pragmatic features, even granting that ordinary explanation does? To keep us on our toes, I'll discuss the second of these first.

Any defender of the connection between grounding and metaphysical explanation must have one of two things in mind. First, they might think that metaphysical explanation is like ordinary explanation, but in some kind of metaphysical context. Thus, the better understood notion of explanation helps elucidate the more problematic notion of grounding. Alternatively, they might think that metaphysical explanation is a special kind of explanation distinct from the ordinary conception.

The problem with this second view is that it is that aspect of metaphysical explanation which is akin to ordinary explanation (for instance, intuitive understanding of terms such as 'because' and 'in virtue of'; formal features such as asymmetry, transitivity, nonmonotonicity and hyperintensionality; and a sense of what it is to convey answers to why-questions) that allows us our intuitive grasp on metaphysical explanation in the first place. Success in arguing that metaphysical explanation is distinct from ordinary forms of explanation means that we lose our grasp on what metaphysical explanation actually is, and so it is very hard to see how the connection between ground and metaphysical explanation could shed light on grounding. The connection between grounding and explanation is only illuminating in so far as the relevant form of explanation is similar enough to our ordinary conception for us to understand it.

If metaphysical explanation is like ordinary explanation but in a metaphysical context, then (assuming we can meet the challenge of 
specifying what this context is) the problem is that metaphysical explanation, like ordinary explanation, will have pragmatic features. What makes for successful metaphysical explanation will depend (to an extent) on features of agents. If the connection between grounding and explanation is as close as (i) in our original triad suggests, then grounding too has pragmatic features. But that straightforwardly contradicts (iii) - grounding relations are supposed to be entirely objective and mind-independent.

So if metaphysical explanation is to elucidate grounding, then it can't be a form of explanation entirely distinct from our ordinary conception. But if it's like our ordinary conception, then it seems that metaphysical explanation, and hence grounding, has agentrelative features. Perhaps we can escape this troubling consequence by pressing the second of the points with which we began our discussion of metaphysical explanation: a distinction between explanation and successful explanation.

Defenders of the causal theory of explanation (for example, Lewis I986) claim that explaining some event involves citing a portion of its causal history. Successfully explaining some event involves citing a relevant portion of that history, where relevance varies with context. Can't we then say that the entire grounding chain of some entity metaphysically explains it, and avoid having to say that metaphysical explanation has any pragmatic features? Perhaps, but this proposal won't give us what we need to maintain that we can look to explanation to tell us about grounding.

Defenders of the grounding-explanation link think that by reflecting on what metaphysically explains what, we can find out what grounds what. But all that is apparent to us is successful explanation. If we are to appeal to features of explanation to elucidate grounding, it must be the features of successful explanation that we appeal to (since these are the ones we are aware of). But as we have seen, what it is for an explanation to be successful varies with context, and so any useful connection between grounding and metaphysical explanation is a connection between grounding and an agent-relative notion of explanation. Attempts to reject the first horn of our dilemma are unsuccessful: metaphysical explanation does have agent-relative features. 


\section{III}

Second Horn: The Grounding-Explanation Connection. It is unappealing to deny that metaphysical explanation has some agentrelative features, and so to resolve the grounding-explanation problem we must now consider re-evaluating the purported link between grounding and metaphysical explanation. I take it that there are broadly three ways to understand the connection. First, we can think of metaphysical explanations as tracking grounding relations. Second, we might think that grounding relations have a closer connection to explanation, such that the grounding relation just is an explanatory relation. Both accounts of the grounding-explanation connection can find support in the literature. Alternatively, we can deny that grounding and explanation are closely connected at all. I'll return to this third option at the end of the section.

Take the first view. Audi (20I2, pp. I I9-20) insists that 'grounding is not a form of explanation, even though it is intimately connected with explanation ... an explanation ... is something you can literally know; a grounding relation is something you can merely know about'. A metaphysical explanation might be a proposition expressing the grounding relation, but it is not the relation itself.

We rely on intuitions about what explains what in order to tell us what grounds what. Furthermore, we rely on what we know about explanation (formal features and so on) to give us a characterization of grounding. If metaphysical explanations merely track grounding relations, then our reliance on features of explanation for our understanding of grounding is troubling. There is far more scope for error in our judgements about grounding than we ought to consider acceptable. There is an epistemic gap between judgements of ground and judgements of explanation that is not present if grounding just is a form of explanation, and it is especially troubling given the limited supply of alternative methods for discovering facts about grounding. Tracking conceptions of metaphysical explanation rob us of the special insight into grounding that metaphysical explanation has been supposed to provide.

In response to such concerns, a defender of the tracking conception of the link might stress that the grounding-explanation connection is very tight. Perhaps there is something like a necessary connection between grounding and metaphysical explanation, so that whenever there is a grounding relation there will be a 
metaphysical explanation that corresponds to it. But in so tightening the link, other difficulties arise. These are held in common with those who think grounding just is an explanatory relation.

One of the main problems we encounter was discussed in the previous section: the agent-relativity of metaphysical explanation infects grounding, contradicting its assumed mind-independence and objectivity. A second problem is epistemic, and concerns the nature of the grounding relation.

In the grounding literature, appeals to the grounding-explanation connection are often made in order to specify formal features of the grounding relation, such as irreflexivity, asymmetry, transitivity, hyperintensionality and non-monotonicity (see, for example, Rosen 20IO; Raven 20I3, p. 689). Our understanding of the nature of the grounding relation is coloured by our understanding of the nature of metaphysical explanation. A tight conception of the grounding-explanation connection means we cannot untangle agent-relative features from the rest.

Consider the common contention that grounding is asymmetric because explanation is asymmetric (Audi 20I2, p. I02; Lowe 20I0). Symmetric explanations are considered bad explanations because they fail to provide us with any new information. It might be, however, that some cases of symmetrical grounding ought to be considered quite plausible (see Thompson 2016). If it were true that symmetries are ruled out of explanations on epistemic grounds, but there are cases of symmetrical grounding, then the epistemic features of explanation would be influencing our theorizing about grounding in a way that led us to believe false things about the grounding relation - that it is symmetric rather than non-symmetric.

All of this suggests the possibility of severing the link between grounding and explanation, and maintaining that the two concepts are independent and distinct (though they may of course be similar in various respects). The upshot of this would be that it would not be safe to infer anything about the character of grounding relations from that of explanation, and that any appeal to our intuitive sense of what is explanatory in a given context ought to lend little credence to our judgements about grounding.

The major problem with this strategy is that it robs us of one of the most prominent approaches to making sense of the grounding relation. As we are now well aware, a crucial way to understand grounding is via its connection to explanation. If the connection is 
severed, the notion of grounding is once more rendered obscure. Without the grounding-explanation link, our understanding of grounding must come from other sources that do not make appeal to the explanatory character of grounding. ${ }^{1}$

Conclusion. Not much attention has been paid to the nature of metaphysical explanation in the literature. Appeals to metaphysical explanation are made in order to shed light on the notion of grounding, but investigation into the nature of metaphysical explanation uncovers the grounding-explanation problem-a tension between our attitude to explanation, realism about grounding, and the purported link between grounding and explanation. To attempt to solve the problem, we held fixed the thesis that grounding is mindindependent and grappled with the resultant dilemma. Since we found no way to blunt either horn, the only thing left to do is to reject the assumption of the mind-independence of grounding with which we began. ${ }^{2}$

\section{Department of Philosophy University of Southampton Avenue Campus Highfield Southampton SOI7 IBF $\mathrm{UK}$ n.m.thompson@soton.ac.uk}

\section{REFERENCES}

Audi, Paul 20I 2: 'A Clarification and Defense of the Notion of Grounding'. In Correia and Schnieder 20I 2, pp. IOI-2I.

\footnotetext{
${ }^{1}$ Such attempts have been made, but they are beyond the scope of this paper. In any case, without the grounding-explanation connection, the project of making grounding intelligible is severely damaged.

2 Thanks to Darragh Byrne, Nicholas K. Jones, Penelope Mackie, Stefan Roski, Alexander Skiles, Pekka Väyrynen and Alastair Wilson, audiences in Barcelona, Birmingham and Hamburg, and at the 2015 SWIP workshop in York and the 2015 Joint Session in Warwick.
} 
Clark, Michael, and David Liggins 20I2: 'Recent Work on Grounding'. Analysis, 72(4), pp. 8I 2-23.

Correia, Fabrice, and Benjamin Schnieder (eds.) 20I2: Metaphysical Grounding: Understanding the Structure of Reality. Cambridge: Cambridge University Press.

deRosset, Louis 2010: 'Getting Priority Straight'. Philosophical Studies, I49(I), pp. 73-97.

Fine, Kit 200I: 'The Question of Realism'. Philosophers' Imprint, I(I), pp. I-30.

20I2: 'A Guide to Ground'. In Correia and Schnieder 20I2, pp. 37-80.

Lewis, David 1986: 'Causal Explanation'. In his Philosophical Papers, vol. II $=$ pp. 2I 4-40. Oxford: Oxford University Press.

Lowe, E. J. 20Iо: 'Ontological Dependence'. In Edward N. Zalta (ed.), The Stanford Encyclopedia of Philosophy (Spring 2010 Edition). http://plato. stanford.edu/archives/spr2oro/entries/dependence-ontological/.

Raven, Michael 20I3: 'In Defense of Ground'. Australasian Journal of Philosophy, 90(4), pp. 687-70I.

Rosen, Gideon 2010: 'Metaphysical Dependence: Grounding and Reduction'. In Bob Hale and Avid Hoffman (eds.), Modality: Metaphysics, Logic, and Epistemology, pp. I09-36. Oxford: Oxford University Press.

Schaffer, Jonathan 2009: 'On What Grounds What'. In David J. Chalmers, David Manley and Ryan Wasserman (eds.), Metametaphysics: New Essays on the Foundations of Ontology, pp. 347-83. Oxford: Oxford University Press.

Thompson, Naomi 2016: 'Metaphysical Interdependence'. In Mark Jago (ed.), Reality Making, pp. 39-56. Oxford: Oxford University Press. 